Int. J. of The Soc. of Mat. Eng. for Resources Vol. 5 No. $1 \quad 80 \sim 90$ (1997)

\title{
Original
}

\section{A Method for Hydrodynamic Characteristics of a Wing in a Shear Flow}

\author{
by

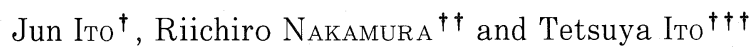

\begin{abstract}
In order to clarify the hydrodynamic characteristics of a wing in a shear flow with arbitrary velocity distribution, an eigenvalue problem of SturmLeouville type holding along the span of the wing is numerically solved and the resulting numerical solution is matched to the analytical solution of the integral equation holding in a plane normal to the span.

This is an example of a "quasi-analytical method".
\end{abstract}

\begin{abstract}
Key Word: Wing, Shear Flow, Hydrodynamic Characteristic, Quasi-analytical method
\end{abstract}

\section{Introduction}

Shear flow problems of a cavitating wing spanning two parallel plane walls are fundamentally important for engineers to perform characteristic analysis and rational design of the high speed hydraulic machinery of axial flow type. However, because there didn't exist any examples treating of the problems in the past, the authors have been studying the analytical methods ${ }^{(1-6)}$ for hydrodynamic characteristics.

In this paper, by introducing a numerical method into a part of the analytical method, a quasianalytical method is proposed, which is easily applicable to the flow with an arbitrary distribution of main stream velocity and is also adaptable, without changing, to the flow with cavitation. That is, this method is directly available to a high speed real flow in engineering.

There are both strength and weakness both in an analytical method to get analytical solutions and in full numerical methods such as finite difference method, finite element method and boundary element method. The article ${ }^{(7)}$ by H. Ohashi concerning this concept strongly attracts us to an analytical method. The authors, admitting the merits that there are in analytical methods, consider the quasi-analytical method as effective which can remove a limit of the anaytical method, and consider it as not effective easily to prefer a full numerical method to an analytical method, even if the problems are complicated.

Received August 31, 1996

$\dagger$ Department of Mechanical Engineering, Akita National College of Technology, Akita City, 011, Japan

$\dagger \dagger$ Department of Applied Mathematics, Tsuruoka National College of Technology, Tsuruoka City, 997, Japan

$\dagger \dagger \dagger$ Department of Information System, Akita Prefectural Techno-school, Akita City, 011, Japan 
Concretely mentioning the quasi-analytical method proposed in this paper, an eigenvalue problem of Sturm-Leouville type holding along the span of the wing is numerically solved and the resulting numerical solution is matched to the analytical solution of the integral equation holding in a plane normal to the span.

As there is room for examination in the aspect of accuracy, we are to improve in it, however.

It seems that the present method is also effective as a means which can estimate the three dimensional effect which is inevitable in the experiment of two dimensional hydrofoil.

\section{Equation of Motion, Boundary Conditions and Solutions ${ }^{(8)}$}

As shown in figure 1, a shear flow which is the fully developed boundary layer between parallel plane walls goes around a wing spanning the walls from infinite upstream in the $\mathrm{x}$ direction. Flow is assumed to be inviscid and incompressible. From the Euler's equation of motion and the equation of continuity, the following equation with respect to disturbance pressure $p$ is derived :

$$
\frac{\partial^{2} p}{\partial x^{2}}+U^{2} \frac{\partial}{\partial y}\left(\frac{1}{U^{2}} \frac{\partial p}{\partial y}\right)+\frac{\partial^{2} p}{\partial z^{2}}=0
$$

For boundary conditions, the disturbance pressure $\mathrm{p}$ is zero at infinity, viz.

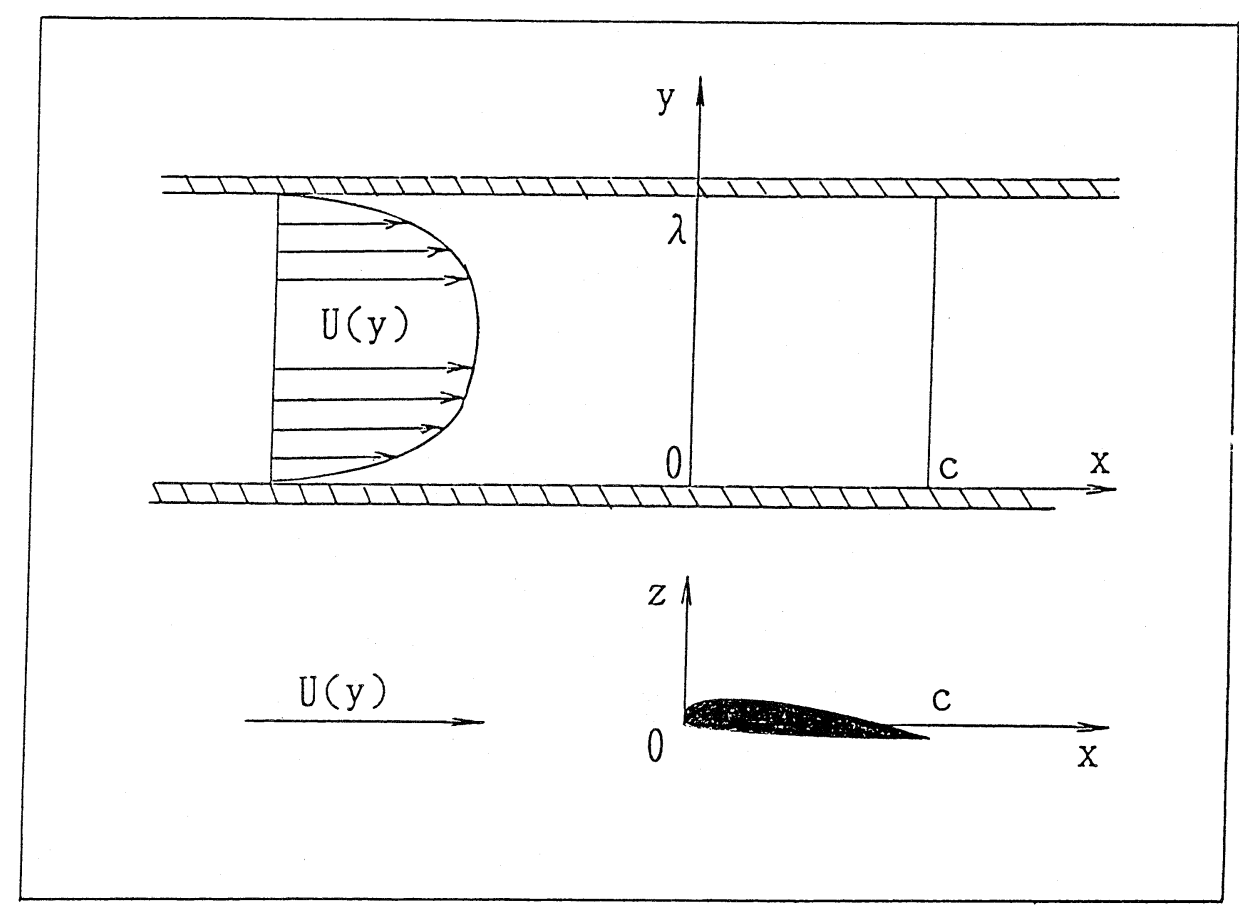

Figure 1. A Wing in a Shear Flow 


$$
\mathrm{p}=0 \quad \text { as } \sqrt{\mathrm{z}^{2}+\mathrm{x}^{2}} \rightarrow \infty
$$

On the plane walls, since the pressure gradient normal to the wall is zero,

$$
\frac{\partial \mathrm{p}}{\partial \mathrm{y}}=0 \quad \text { at } \quad \mathrm{y}=0, \lambda
$$

On the wing surface, from tangential flow condition,

$$
\frac{\mathrm{w}(\mathrm{x}, \mathrm{y}, 0)}{\mathrm{U}(\mathrm{y})}=\frac{\mathrm{d}}{\mathrm{dx}} \mathrm{z}_{\mathrm{f}}(\mathrm{x}) \quad \text { in } 0<\mathrm{x}<\mathrm{c}, 0<\mathrm{y}<\lambda,
$$

where $\mathrm{w}$ is the $\mathrm{z}$-component of disturbance velocity, $z_{\mathrm{f}}$ the wing section shape, $\lambda$ the wing span length, and $\mathrm{c}$ the wing chord length.

When an elementary solution is assumed to be that of separation of variable as

$$
\frac{\mathrm{p}}{\rho}=\mathrm{Y}(\mathrm{y}) \mathrm{P}(\mathrm{x}, \mathrm{z})
$$

the general solution becomes

$$
\frac{\mathrm{p}}{\rho}=\sum_{\mathrm{n}=1}^{\infty} \mathrm{F}(\mathrm{kn}) \mathrm{Y}(\mathrm{y} ; \mathrm{kn}) \mathrm{P}(\mathrm{x}, \mathrm{z}, ; \mathrm{kn})
$$

where $\rho$ is the fluid density, kn the separation constant and $F(k n)$ the spectrum.

The function $P(x, z ; k n)$ is given by

$$
\mathrm{P}(\mathrm{x}, \mathrm{z} ; \mathrm{kn})=\frac{1}{2 \pi} \int_{0}^{\mathrm{c}} \Sigma(\xi ; \mathrm{kn}) \frac{\partial}{\partial \mathrm{x}} \mathrm{K}_{0}(\mathrm{r}) \mathrm{d} \xi+\frac{1}{2 \pi} \int_{0}^{\mathrm{c}} \Gamma(\xi ; \mathrm{kn}) \frac{\partial}{\partial \mathrm{z}} \mathrm{K}_{0}(\mathrm{r}) \mathrm{d} \xi
$$

where $\Gamma$ and $\Sigma$ are non-dimensional quantities being analogous to the vortex and source in uniform flow which coincide with two dimensional ones, and $\mathrm{K}_{0}$ is the modified Bessel function of the second kind, of order zero, and

$$
\mathrm{r}=\mathrm{kn}\left\{\mathrm{z}^{2}+(\mathrm{x}-\xi)^{2}\right\}^{\frac{1}{2}}
$$

On the other hand, the function $\mathrm{Y}(\mathrm{y} ; \mathrm{kn})$ in equation (2.6) is the solution of the boundary value problem :

$$
\begin{array}{ll}
\frac{d}{d y}\left\{\frac{1}{U(y)^{2}}\right. & \left.\frac{d Y}{d y}\right\}+k^{2} \frac{Y}{U(y)^{2}}=0 \\
\frac{d Y}{d y}=0 & \text { at } y=0, \lambda
\end{array}
$$

\section{Analysis of Spanwise direction}

\section{1 Eigenvalue Problem}

By considering the symmetry of the flow field, to reduce the numerical calculation, the problem for the spanwise analysis is rewritten as 


$$
\begin{aligned}
& \frac{d^{2} Y}{d y^{2}}-\left\{\frac{2}{U(y)} \quad \frac{d}{d y} \quad U(y)\right\} \frac{d Y}{d y}+k^{2} Y=0 \\
& \frac{d Y}{d y}=0 \quad \text { at } \quad y=0 \\
& \frac{d Y}{d y}=0 \quad \text { at } \quad y=\frac{\lambda}{2} .
\end{aligned}
$$

This boundary value problem to solve the equation (3. 1) on boundary conditions (3. 2) and (3. 3) is a Sturm-Liouville type of eigenvalue problem, and there are an infinite number of eigenvalues $\mathrm{kn}(>0)$, to each of which an eigenfunction $\mathrm{Y}(\mathrm{y} ; \mathrm{kn})$ corresponds, so that

$$
\int_{0}^{\frac{\lambda}{2}} \frac{\mathrm{Y}(\mathrm{y} ; \mathrm{kn}) \mathrm{Y}(\mathrm{y} ; \mathrm{kr})}{\mathrm{U}(\mathrm{y})^{2}} d y=0 \quad(\mathrm{n} \neq \gamma) .
$$

If these functions are normalized as

$$
\frac{2}{\lambda} \int_{0}^{\frac{\lambda}{2}} \frac{\mathrm{Y}(\mathrm{y} ; \mathrm{kn})^{2}}{\mathrm{U}(\mathrm{y})^{2}} \mathrm{dy}=1,
$$

the spectrum $F(\mathrm{kn})$ in equation (2.6) can be determined as

$$
\begin{aligned}
& \mathrm{F}(\mathrm{kn})=\frac{2}{\lambda} \int_{0}^{\frac{\lambda}{2}} \mathrm{Y}(\mathrm{y} ; \mathrm{kn}) \mathrm{dy} \\
& \mathrm{F}(0)=\mathrm{Y}(\mathrm{y} ; 0)=\left\{\frac{2}{\lambda} \int_{0}^{\frac{\lambda}{2}} \frac{1}{\mathrm{U}(\mathrm{y})^{2}} \mathrm{dy}\right\}^{-\frac{1}{2}} .
\end{aligned}
$$

\section{2 Difference Equation}

As shown in figure 2, the range of independent variable in question (that is $\lambda / 2$ ) is subdivided into $n$ equal parts, the length of the subdivision is denoted by $h, Y(y)$ at $y_{i}=y_{0}+i h$ by $Y_{i}$, and $\mathrm{U}(\mathrm{y})$ by $\mathrm{U}_{\mathrm{i}}$. In order that the equation (3.1) is discretized by finite difference, the approximations

$$
\begin{aligned}
& \frac{d^{2} Y}{d y^{2}} \fallingdotseq \frac{Y_{i-1}-2 Y_{i}+Y_{i+1}}{h^{2}} \\
& \frac{d U}{d y} \fallingdotseq \frac{U_{i+1}-U_{i-1}}{2 h} \\
& \frac{d Y}{d y} \fallingdotseq \frac{Y_{i+1}-Y_{i-1}}{2 h}
\end{aligned}
$$

are substituted into the equation (3. 1), which is reduced to

$$
Y_{i-1}-2 Y_{i}+Y_{i+1}-\frac{U_{i-1}-U_{i+1}}{2 U_{i}}\left(Y_{i-1}-Y_{i+1}\right)+k^{2} h^{2} Y_{i}=0 .
$$




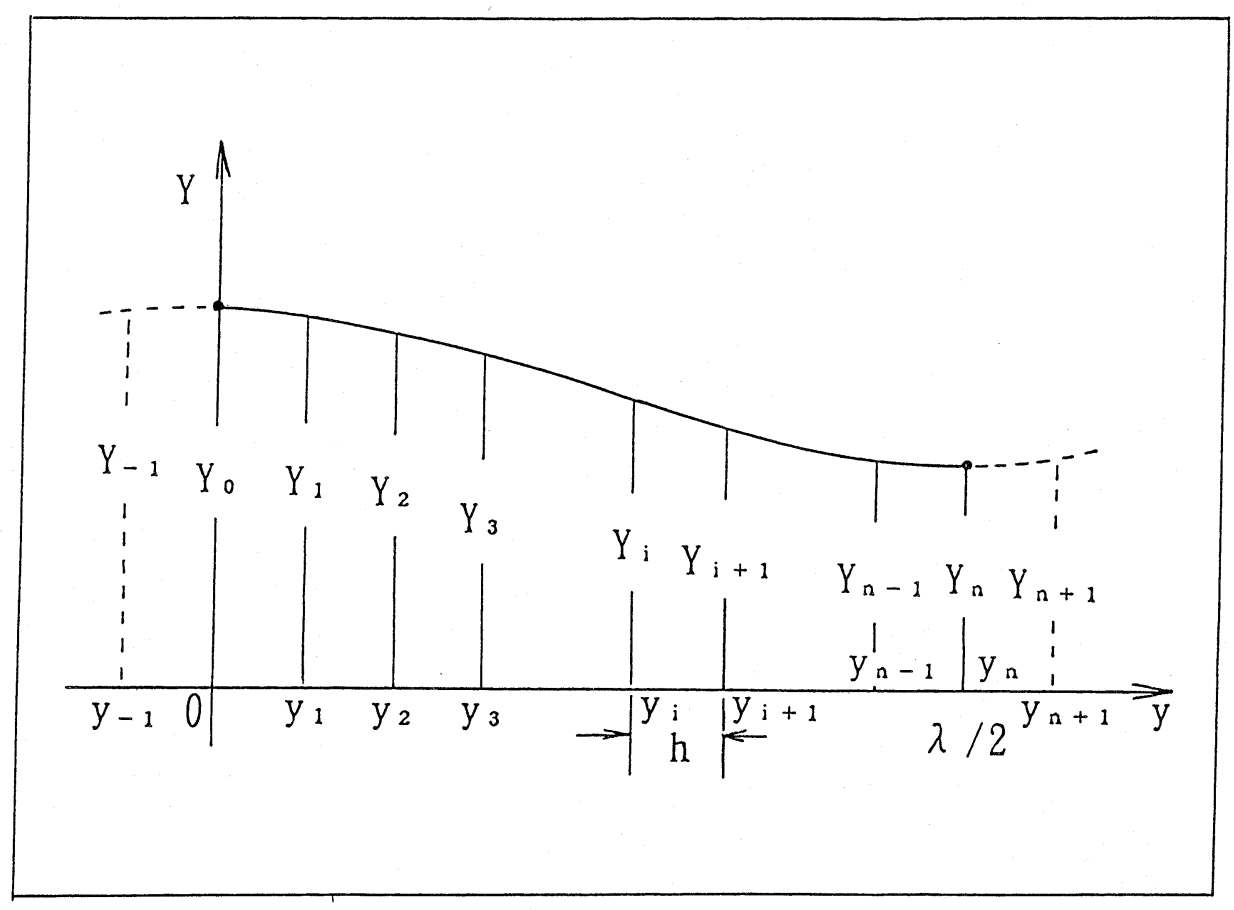

Figure 2. Division

And the boundary conditions (3.2) and (3.3) become respectively

$$
\begin{aligned}
& \mathrm{Y}_{1}-\mathrm{Y}_{-1} \fallingdotseq 0 \\
& \mathrm{Y}_{\mathrm{n}+1}-\mathrm{Y}_{\mathrm{n}-1} \fallingdotseq 0
\end{aligned}
$$

In the above symmetrical difference forms, there are no restrictions on the velocity distribution of the main stream, which can be therefore arbitrarily given, and it is strong point in the present method.

Here, as an example, Kàrma'n-Prandtl's one seventh power velocity distribution law holding in the turbulent boundary layer is used and is expressed as follows, from which the discrete values are taken out for numerical calculation,

$$
\begin{aligned}
& \mathrm{U}(\mathrm{y})=\mathrm{U}\left(\frac{\lambda}{2}\right)\left(\frac{2 \mathrm{y}}{\lambda}\right)^{\frac{1}{t}} \text { in } 0<\mathrm{y}<\frac{\lambda}{2} \\
& \mathrm{U}(\mathrm{y})=\mathrm{U}\left(\frac{\lambda}{2}\right)\left\{\frac{2(\lambda-\mathrm{y})}{\lambda}\right\}^{\frac{1}{7}} \text { in } \frac{\lambda}{2}<\mathrm{y}<\lambda .
\end{aligned}
$$

After differentiating the equation (3.14) with respect to y, if the resulting derivative is multiplied by $2 / \mathrm{U}(\mathrm{y})$, 


$$
\frac{2}{U(y)} \frac{d}{d y} U(y)=\frac{2}{7 y} .
$$

Therefore the equation (3.1) becomes

$$
\frac{d^{2} Y}{d y^{2}}-\frac{2}{7 y} \frac{d Y}{d y}+k n^{2} Y=0
$$

The difference form of the equation (3.17) is, from equations (3.8) and (3.10),

$$
Y_{i-1}-2 Y_{i}+Y_{i+1}+\frac{h}{7 y_{i}}\left(Y_{i-1}-Y_{i+1}\right)+h^{2} k n^{2} Y_{i}=0
$$

where by putting

$$
\mathrm{y}_{\mathrm{i}}=\mathrm{y}_{0}+\mathrm{ih}=\mathrm{ih}
$$

the equation (3.18) is reduced to, after some reduction,

$$
\left(1+\frac{1}{7 \mathrm{i}}\right) \mathrm{Y}_{\mathrm{i}-1}+\left(\mathrm{h}^{2} \mathrm{kn}^{2}-2\right) \mathrm{Y}_{\mathrm{i}}+\left(1-\frac{1}{7 \mathrm{i}}\right) \mathrm{Y}_{\mathrm{i}+1}=0 \text {. }
$$

This is the difference equation corresponding to the differential equation (3. 17).

\section{3 Characteristic Equation}

By putting

$$
\mathrm{K}=\mathrm{h}^{2} \mathrm{kn}^{2} \text {, }
$$

the equation (3.20). becomes

$$
-\left(1+\frac{1}{7 \mathrm{i}}\right) \mathrm{Y}_{\mathrm{i}-1}+(2-\mathrm{K}) \mathrm{Y}_{\mathrm{i}}-\left(1-\frac{1}{7 \mathrm{i}}\right) \mathrm{Y}_{\mathrm{i}+1}=0 .
$$

Derivative of the equation (3.14) with respect to $\mathrm{y}$ is infinite at $\mathrm{y}=0$, and this matter causes the so-called "paradox" that the shear stress becomes infinite on the wall $(y=0)$. Further the second term of the equation (3.17) is in indefinite form, so that this equation does not hold at $\mathrm{y}=$ 0 . To keep away from these difficulties, it is assumed that $\mathrm{i}=0$ corresponds not to $\mathrm{y}=0$ but to $\mathrm{y}$ $=\varepsilon(>0,<<\mathrm{h})$ and that $\frac{d \mathrm{Y}}{\mathrm{dy}}=0$ still holds at $\mathrm{y}=\varepsilon$. However small $\varepsilon$ may be, as far as it is finite, the second term of the equation (3.17) becomes zero, so that the equation (3. 22) at $\mathrm{i}=0$ is reduced to rational form. Including this form, the following $\mathrm{n}$ simultaneous algebraic equations are derived from the equation (3. 22).

$$
\begin{aligned}
& (2-\mathrm{K}) \mathrm{Y}_{0}-2 \mathrm{Y}_{1}=0 \\
& -\frac{8}{7} \mathrm{Y}_{0}+(2-\mathrm{K}) \mathrm{Y}_{1}-\frac{6}{7} \mathrm{Y}_{2}=0 \\
& -\frac{15}{14} \mathrm{Y}_{1}+(2-\mathrm{K}) \mathrm{Y}_{2}-\frac{13}{14} \mathrm{Y}_{3}=0
\end{aligned}
$$




$$
\begin{aligned}
& -\frac{7 j+1}{7 j} Y_{j-1}+(2-K) Y_{j}-\frac{7 j-1}{7 j} Y_{j+1}=0 \\
& -\frac{7(n-1)+1}{7(n-1)} Y_{n-2}+(2-K) Y_{n-1}-\frac{7(n-1)-1}{7(n-1)} Y_{n}=0 \\
& -2 Y_{n-1}+(2-K) Y_{n}=0
\end{aligned}
$$

where the boundary conditions (3.12), (3.13) are introduced.

For $Y_{i}$ to have a value other than zero, a necessary and sufficient condition for solution is that the determinant of the coefficient matrix of the set of homogeneous equations (3.23) equals zero, and so the following characteristic equation holds.

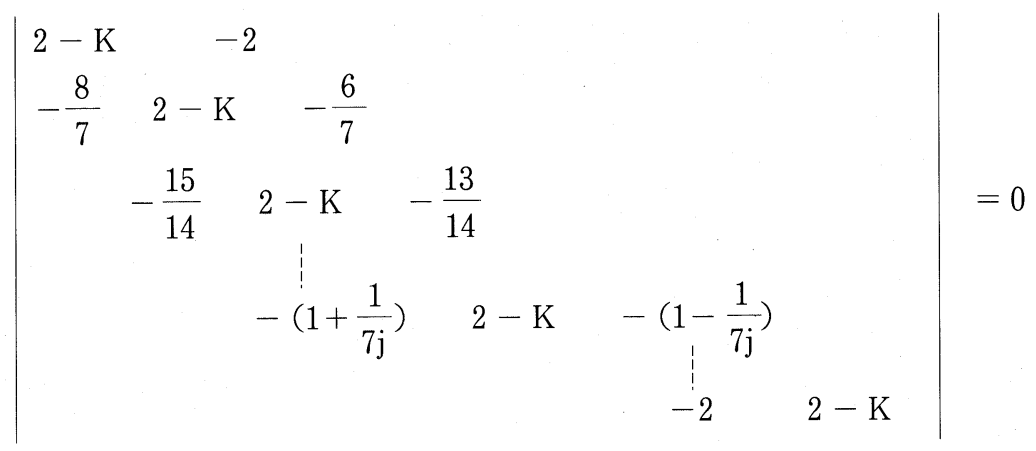

To solve the characteristic equation (3. 24) the QR method ${ }^{(9)}$ is used, K's are determined and kn's too from the relation (3. 21).

\section{4 Eigenfunction}

For determining $Y_{j}$, the following equations obtained by modifying the equation (3.23) can be used.

$$
\begin{aligned}
& Y_{1}=\frac{1}{2} K^{\prime} Y_{0} \\
& Y_{j+1}=-\frac{1}{B}\left(A Y_{j-1}+K^{\prime} Y_{j}\right)(j=1,2, \cdots \cdots, n-2) \\
& Y_{n}=\frac{2}{K^{\prime}} Y_{n-1}
\end{aligned}
$$

where

$$
\begin{aligned}
& K^{\prime}=2-K \\
& A=-\frac{7 j+1}{7 j} \\
& B=-\frac{7 j-1}{7 j} .
\end{aligned}
$$

Next, the normalization of the eigenfunction should be performed. $\mathrm{Y}_{0}=1$ is put, all $\mathrm{Y}_{\mathrm{j}}$ 's are determined from the equations (3. 25), (3. 26) and (3. 27), and the integral of equation (3. 5) is 
executed by using them, whose value is denoted by S. The function $\mathrm{Y}(\mathrm{y} ; \mathrm{kn})$ is defined anew by regarding $\mathrm{Y}(\mathrm{y} ; \mathrm{kn}) / \mathrm{S}^{\frac{1}{2}}$ as it, so that the normalizing condition (3.5) is satisfied.

\section{Problem in Wing Section Plane ${ }^{(6)}$}

\section{1 Integral Equation}

From equation (2.7) the pressure function $\mathrm{P}(\mathrm{x}, \pm 0 ; \mathrm{kn})$ and the non-dimensional disturbance velocity $\mathrm{w}_{\mathrm{n}}(\mathrm{x}, \pm 0 ; \mathrm{kn})$ become

$$
\begin{aligned}
& \mathrm{P}(\mathrm{x}, \pm 0 ; \mathrm{kn})=\mp \frac{1}{2} \Gamma(\mathrm{x}, \mathrm{kn})-\frac{1}{2 \pi} \int_{0}^{\mathrm{c}} \sum(\xi ; \mathrm{kn}) \frac{1}{\mathrm{x}-\xi} \mathrm{d} \xi-\frac{1}{2 \pi} \mathrm{kn} \int_{0}^{\mathrm{c}} \sum(\xi ; \mathrm{kn}) \\
& \times \operatorname{sgn}(\mathrm{x}-\xi) \mathrm{R}_{1}(\mathrm{kn}|\mathrm{x}-\xi|) \mathrm{d} \xi \\
& \mathrm{w}_{\mathrm{n}}(\mathrm{x}, \pm 0 ; \mathrm{kn})= \pm \frac{1}{2} \sum(\mathrm{x} ; \mathrm{kn})-\frac{1}{2 \pi} \int_{0}^{\mathrm{c}} \Gamma(\xi ; \mathrm{kn}) \frac{1}{\mathrm{x}-\xi} \mathrm{d} \xi-\frac{1}{2 \pi} \mathrm{kn} \int_{0}^{\mathrm{c}} \Gamma(\xi ; \mathrm{kn}) \\
& \quad \times \operatorname{sgn}(\mathrm{x}-\xi)\left\{\mathrm{R}_{1}(\mathrm{kn}|\mathrm{x}-\xi|)+\int_{0}^{\mathrm{kn}|\mathrm{x}-\xi|} \mathrm{K}_{0}(\mathrm{t}) \mathrm{dt}\right\} \mathrm{d} \xi \\
& \quad-\frac{1}{4} \mathrm{kn}_{0}^{\mathrm{c}} \Gamma(\mathrm{x} ; \mathrm{kn}) \mathrm{dx}
\end{aligned}
$$

where $R_{1}(z)=K_{1}(z)-1 / z$, in which $K_{1}$ is the modified Bessel function of the second kind, of order one. Camber line of the wing is denoted by $\mathrm{z}_{\mathrm{c}}(\mathrm{x})$, then

$$
\frac{\mathrm{d}}{\mathrm{dx}} \mathrm{z}_{\mathrm{c}}(\mathrm{x})=\frac{1}{2}\left\{\mathrm{w}_{\mathrm{n}}(\mathrm{x},+0 ; \mathrm{kn})+\mathrm{w}_{\mathrm{n}}(\mathrm{x},-0 ; \mathrm{kn})\right\}
$$

and so

$$
\begin{aligned}
& \frac{d}{d x} z_{c}(x)=-\frac{1}{2 \pi} \int_{0}^{c} \Gamma(\xi ; k n) \frac{1}{x-\xi} d \xi-\frac{1}{2 \pi} \operatorname{kn} \int_{0}^{c} \Gamma(\xi ; k n) \operatorname{sgn}(x-\xi) \\
& \times\left\{R_{1}(k n|x-\xi|)+\int_{0}^{\mathrm{kn}|x-\xi|} K_{0}(t) d t\right\} d \xi-\frac{1}{4} k n \int_{0}^{c} \Gamma(x ; k n) d x
\end{aligned}
$$

holds. Equation (4.4) is the integral equation with unknown function $\Gamma(\mathrm{x} ; \mathrm{kn})$ and also governing one used for analyzing the flow in x-z plane.

\section{2 Method of Solution of Integral Equation}

To solve the integral equation (4.4), unknown function $\Gamma(\mathrm{x} ; \mathrm{kn})$ is expanded as

$$
\Gamma(\mathrm{x} ; \mathrm{kn})=\mathrm{A}_{0}(\mathrm{kn}) \cot \frac{\phi}{2}+\sum_{\mathrm{m}=1}^{\infty} \mathrm{A}_{\mathrm{m}}(\mathrm{kn}) \sin \mathrm{m} \phi, \mathrm{x}=\frac{\mathrm{c}}{2}(1-\cos \phi) .
$$

Substituting the equation (4.5) into equation (4.4), the following algebraic equation is obtained.

$$
\frac{\mathrm{d}}{\mathrm{dx}} \mathrm{z}_{\mathrm{c}}(\mathrm{x})=\mathrm{A}_{0}(\mathrm{kn})\left(-\frac{1}{2}\left(1+\frac{1}{4} \pi \mathrm{ckn}\right)-\frac{1}{2 \pi} \mathrm{kn} \int_{0}^{\mathrm{c}} \operatorname{sgn}(\mathrm{x}-\xi)\left\{\mathrm{R}_{1}(\mathrm{kn}|\mathrm{x}-\xi|)\right.\right.
$$




$$
\begin{aligned}
& \left.\left.+\int_{0}^{\mathrm{kn}|\mathrm{x}-\xi|} \mathrm{K}_{0}(\mathrm{t}) \mathrm{dt}\right\} \cot \frac{\phi}{2} \mathrm{~d} \xi\right]+\mathrm{A}_{1}(\mathrm{kn})\left(\frac{1}{2}\left(\cos \phi-\frac{1}{8} \pi \mathrm{ckn}\right)\right. \\
& \left.-\frac{1}{2 \pi} \mathrm{kn} \int_{0}^{\mathrm{c}} \operatorname{sgn}(\mathrm{x}-\xi)\left\{\mathrm{R}_{1}(\mathrm{kn}|\mathrm{x}-\xi|)+\int_{0}^{\mathrm{kn}|\mathrm{x}-\xi|} \mathrm{K}_{0}(\mathrm{t}) \mathrm{dt}\right\} \sin \phi \mathrm{d} \xi\right) \\
& +\sum_{\mathrm{m}=2}^{\infty} \mathrm{A}_{\mathrm{m}}(\mathrm{kn})\left[\frac{1}{2} \cos \mathrm{m} \phi-\frac{1}{2 \pi} \mathrm{kn} \int_{0}^{\mathrm{c}} \operatorname{sgn}(\mathrm{x}-\xi)\left\{\mathrm{R}_{1}(\mathrm{kn}|\mathrm{x}-\xi|)\right.\right. \\
& \left.\left.+\int_{0}^{\mathrm{kn}|\mathrm{x}-\xi|} \mathrm{K}_{0}(\mathrm{t}) \mathrm{dt}\right\} \sin \mathrm{m} \phi \mathrm{d} \xi\right]+\cdots \cdots
\end{aligned}
$$

Since the pivotal point is on chord line, three quarter chord theorem is adopted.

That is

$$
\mathrm{x}_{\nu}=\frac{\mathrm{c}}{4 \mathrm{~N}}(4 \nu-1)
$$

where $\mathrm{N}$ is the number of the pivotal point and is 3 .

\section{Characteristics of Wing}

\section{1 Lift Coefficient Distribution}

By spanwisely integrating the pressure difference between upper and lower surfaces of the wing, a local lift in the section plane at any position is

$$
l(y)=\int_{0}^{c}\{p(x, y,-0)-p(x, y,+0)\} d x .
$$

A lift coefficient distribution is defined as

$$
\mathrm{C}_{1}(\mathrm{y})=\frac{2 \mathrm{l}(\mathrm{y})}{\rho \mathrm{U}(\mathrm{y})^{2}} .
$$

Accordingly from equation (2.6), (5.1) and (5.2),

$$
\mathrm{C}_{1}(\mathrm{y})=\frac{1}{2} \frac{\pi}{\mathrm{U}(\mathrm{y})^{2}} \sum_{\mathrm{n}=1}^{\infty} \mathrm{F}(\mathrm{kn}) \mathrm{Y}(\mathrm{y} ; \mathrm{kn})\left\{2 \mathrm{~A}_{0}(\mathrm{kn})+\mathrm{A}_{1}(\mathrm{kn})\right\}
$$

\section{2 Numerical Example}

Figure 3 shows the spanwise lift coefficient distribution for the rectangular wing with flat section. The small black dots are the calculated ones, which disperse and are not smooth. The solid curve is smoothly drawn by roughly taking an average value of these dots. The dashed line shows the two dimensional value $2 \pi$ of the lift coefficient which is divided by attack angle $\alpha$. The difference between the straight line shown by the dashed line and the curved line of spanwise lift coefficient distribution shown by the solid line means the three dimensional effect caused by the nonuniformity of the main stream velocity distribution peculiar to the boundary layer flow.

For the mechanism of this effect the following two matters are principally enumerated : one thing is that the velocity of outer flow layer increases and that of inner one decreases as a result of 


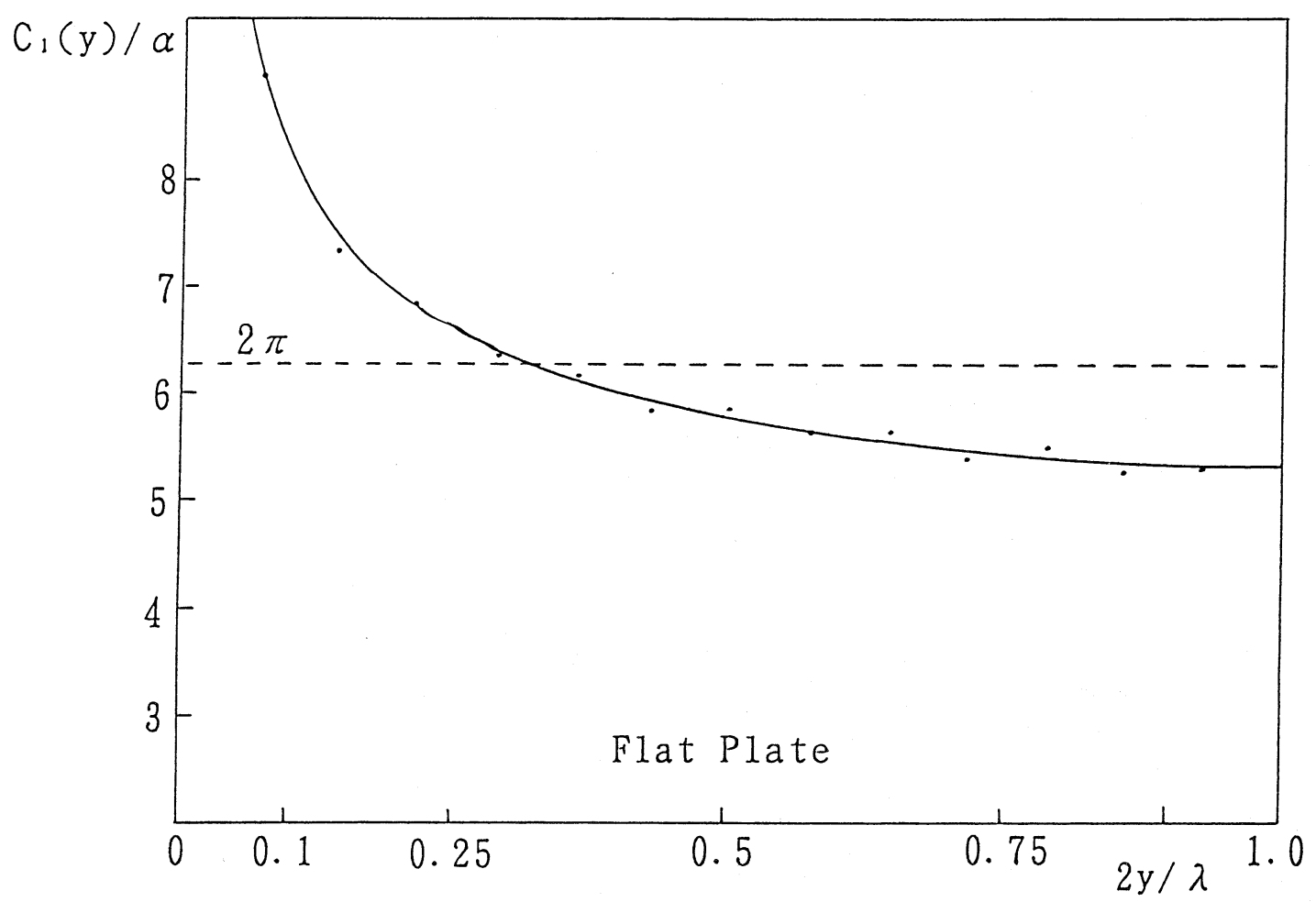

Figure 3. Lift Coefficient Distribution

the deformation of the longitudinal vortex sheet of the main stream, and the other thing is the non-uniformity of the spanwise distribution of the induced velocity resulting from the trailing vortex.

It is clear that the accelerating flow in outer flow layer, which is one of the three dimensional effects, causes the phenomenon of the so-called cavitation or stall near the wall that is at the tip of the wing.

\section{Conclusions}

A quasi-analytical method is developed, and the hydrodynamic characteristic is clarified.

This paper is summerized as follows.

(1) The eigenvalue problem with respect to the spanwise function is formulated by introducing the separation of variable into the equation of motion derived by von Kàrmàn which holds for a wing in a non-uniform flow.

(2) Though the distribution of the main stream velocity can be arbitrarily given, here as an example, that of the boundary layer flow velocity subject to the one seventh power law is assumed, and then the equations in the eigenvalue problem are reduced to the difference equations by discretization.

(3) From applying the difference equation to all the divisional locations and from difference forms 
of the boundary conditions, the characteristic equation is derived, and it is solved by the QR method so that the eigenvalues are determined.

(4) The eigen functions corresponding to the individual eigenvalues are temporarily calculated by using the iterative procedure, and are recalculated in order to satisfy the imposed normalization.

(5) In the plane normal to the span of the wing, the integral equation involving the eigenvalues is derived from the analogy of the acceleration potential method used in the ordinary wing theory, and is reduced to the simultaneous linear algebraic equation, which is solved by Gauss elimination method.

(6) The numerical solutions of the spanwise problem, which are eigenvalue, eigen function and spectrum, are matched to the series solutions analytically obtained in the plane normal to the span of the wing, and then the lift coefficient distribution is shown and the three dimensional effect due to the wall boundary layer is clarified.

\section{Acknowledgement}

This research is supported by the Ministry of Education, Science and Culture, through the Grantin-Aid for scientific research.

\section{References}

(1) Ito, J. \& Narita, A., Trans of JSME, 57-537, pp.1635-1639 (1991.5).

(2) Ito, J., Trans of JSME, 57-543, pp.3783-3787 (1991.11).

(3) Ito, J. et al., Trans of JSME, 59-567, pp.3393-3398 (1993.11).

(4) Ito, J., Trans of JSME, 56-530, pp.2871-2878 (1990.10)

(5) Ito, J., Trans of JSME, 57-536, pp.1289-1296 (1991.4).

(6) Ito, J. et al., Trans of JSME, 56-522, pp.295-300 (1990.2).

(7) Ohashi, H., Jour. of JSME, 87-785, pp.299-303 (1984.4).

(8) Honda, M., Proc.R.Soc., Ser. A. 254, pp.372-394 (1960).

(9) Nakamura, R. et al., Numerical Method and Engineering Problems (FORTRAN), pp.39-53, Morikita Shuppan, (1992). 